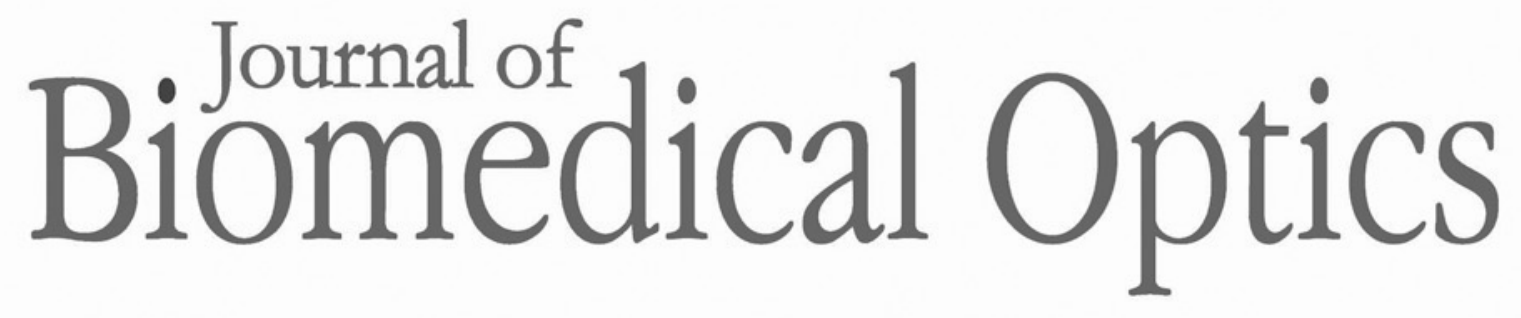

BiomedicalOptics.SPIEDigitalLibrary.org

\title{
A special year for light
}

Lihong V. Wang, Ph.D.

\section{SPIE.}




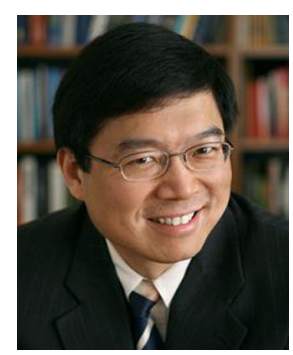

\section{A special year for light}

To celebrate the contribution of optics to humankind, the UN proclaimed 2015 as the International Year of Light and Lightbased Technologies. Light is truly special. The minute optical region of the vast electromagnetic spectrum is the only one that provides molecular information directly. Due to the fundamental importance of molecules to organisms, light plays a unique role in biology and medicine. Interfacing between engineering and biomedicine, biomedical optics will continue to grow in years to come and will find even greater impact in human health.

The Journal of Biomedical Optics has grown with our field by providing an essential platform for information exchange. Its success is attributed to the contributions of our authors, editorial board members, guest editors, reviewers, and staff. In particular, we are grateful to the following top reviewers who reviewed the most papers in the year of 2015:

1. Yasaman Ardeshirpour, U.S. Food and Drug Administration

2. Mohammad Avanaki, Wayne State University

3. Daniel Elson, Imperial College London

4. Daniel Fried, University of California at San Francisco
5. Xiaoxing Han, Massachusetts General Hospital

6. Stephen Kanick, Dartmouth College

7. Bjoern Kemper, University of Muenster

8. Chulhong Kim, POSTECH

9. Robert Nuster, Karl-Franzens-University Graz

10. Amy Oldenburg, The University of North Carolina at Chapel Hill

11. Cynthia Pagba, Georgia Tech

12. Daqing Piao, Oklahoma State University

13. Alexey Popov, University of Oulu

14. Jessica Ramella-Roman, Florida International University

15. Hamideh Salehi, University of Montpellier

16. Tilman Schmoll, Carl Zeiss Meditec

17. Ronald Sroka, Klinikum der University

18. Herbert Stepp, Ludwig Maximilians University

19. Chen $\mathrm{Xu}$, New York City College of Technology

20. Junjie Yao, Washington University in St. Louis

21. Haishan Zeng, British Columbia Cancer Agency Research Centre

22. Dan Zhu, Huazhong University of Science and Technology

As always, I welcome any suggestions for improving JBO and look forward to working with you.

Lihong V. Wang Editor-in-Chief 